\title{
Hyperoxia improves contrast sensitivity in early diabetic retinopathy
}

Department of

Ophthalmology,

Indiana University

School of Medicine,

Indianapolis, USA

A Harris

R P Danis

Department of Physiology and Biophysics, Indiana University School of Medicine,

Indianapolis, USA

A Harris

Augenklinik der Med Fak, RWTH Aachen, Germany

$O$ Arend

$S$ Wolf

Visual Sciences Program, Indiana University School of Optometry,

Bloomington, USA

D Evans

Medical Sciences Program, Indiana University, Bloomington, USA B J Martin

Correspondence to: Alon Harris, $\mathrm{PhD}$

Department of

Ophthalmology, Indian University School of

Medicine, 702 Rotary Circle Indianapolis, IN 46202, USA.

Accepted for publication 9 November 1995

\author{
Alon Harris, Oliver Arend, Ronald P Danis, David Evans, Sebastian Wolf, \\ Bruce J Martin
}

\begin{abstract}
Aim-The cause of vascular and visual pathology in diabetic retinopathy remains unknown. If retinal hypoxia plays a role, then early in the course of diabetes $100 \%$ oxygen breathing should normalise both contrast sensitivity and retinal blood flow. Methods-This hypothesis was tested in 12 diabetic patients with minimal retinopathy who, none the less, exhibited reduced contrast sensitivity $(p=0.003$ versus 12 age and sex-matched controls) and prolonged retinal arteriovenous dye transit ( $p=0.0001$ versus controls).

Results-Isocapnic hyperoxia failed to alter contrast sensitivity in controls, while it significantly improved contrast sensitivity in patients (at $12 \mathrm{cpd} p=0.042$ ) to levels indistinguishable from normal. Individual improvement in contrast sensitivity correlated positively with the severity of the initial defect $(r=+0 \cdot 84$, $p=0.0008)$. Hyperoxia also had haemodynamic effects: it slowed retinal arteriovenous passage of fluorescein dye in controls, but did not further slow this transit time in patients.

Conclusions-These results demonstrate the reversibility of early contrast sensitivity deficits in diabetes mellitus, and support the hypothesis that factors linked to tissue hypoxia initiate both visual and vascular dysfunction in diabetic retinopathy.
\end{abstract}

(Br f Ophthalmol 1996; 80: 209-213)

Diabetes mellitus patients, both with and without visible retinopathy, demonstrate reductions in contrast sensitivity and colour perception, and alterations in the pattern electroretinogram. ${ }^{1-4}$ In patients with advanced disease, visual function losses correlate well with both the level of retinopathy and the extent of retinal oedema. ${ }^{15}$ Further, patients whose retinal oedema regresses after panretinal photocoagulation also show improved contrast sensitivity. ${ }^{6}$ None the less, in patients with early stage diabetes, little is known about how the disease provokes visual loss. In these patients, no visible evidence of damage in the retina is available to explain reductions in visual function.

Among the earliest vascular changes associated with diabetes are vasodilatation and increased retinal blood flow. ${ }^{7}$ These changes have been hypothesised to result from either retinal tissue hypoxia ${ }^{89}$ or from hyperglycaemia pseudohypoxia. ${ }^{7}$ This latter theory holds that hyperglycaemia induced increases in cytosolic NADH/NAD ${ }^{+}$ratio provoke hyperaemia, endothelial damage, and vascular wall sclerosis. ${ }^{7}$ If either of these theories is correct, then in early diabetic retinopathy, before major changes in vessel architecture have occurred, reducing tissue hypoxia (or NADH/NAD ${ }^{+}$ ratios) with hyperoxia should normalise both blood flow and visual function. To test this possibility, in this study we examined in early stage diabetes the simultaneous influence of $100 \%$ oxygen breathing on both visual function (contrast sensitivity) and retinal perfusion (retinal arteriovenous passage time).

\section{Patients and methods}

Twelve insulin dependent diabetic patients and 12 healthy subjects were tested. All participated after signing informed consent to procedures reviewed and approved by a committee for protection of human subjects. The research conformed to the tenets of the Declaration of Helsinki.

DIABETIC SUBJECTS

Patients were recruited from the clinical practice of the Retina Service at the Indiana University Medical Center. All subjects presented for routine ophthalmic screening for diabetic retinopathy. Subjects had had insulin dependent diabetes for at least 5 years and no concurrent systemic illness (for example, hypertension or renal failure). An ophthalmic examination consisting of history, refraction, visual acuity measurement, slit-lamp, and detailed fundus examination was performed on each subject. Fundus examination was performed with a 90 dioptre lens at the slit-lamp and indirect ophthalmoscopy was carried out with a 20 dioptre lens. Visual acuity was $20 / 25$ or better (corrected) in all subjects. The level of retinopathy was estimated in each eye. Only eyes with no detectable retinopathy or with haemorrhages and/or microaneurysms grade Ia or less (by the DRS modified Airlie House classification ${ }^{10}$ ) and no other signs of retinopathy were included.

NORMAL SUBJECTS

Healthy volunteers were recruited from the subject pool at the Indiana University Medical Center; they were chosen so that their mean age and distribution by sex would match that of the diabetic subjects. All normal subjects had $20 / 20$ or better best corrected visual acuity. 


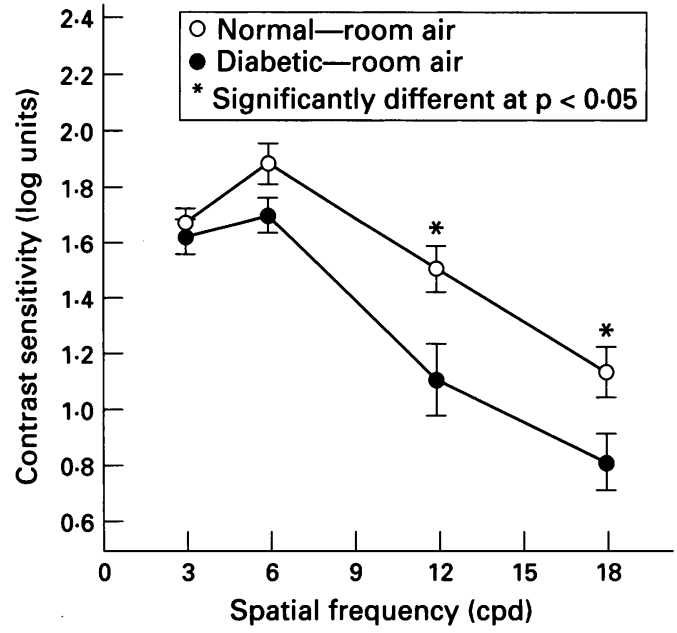

Figure 1 Contrast sensitivity at four spatial frequencies in normals and in diabetics with minimal retinopathy. Diabetics displayed depressed sensitivity at 12 and 18 cycles per degree (asterisks indicate a statistically significant difference $(p=0.003$ at $12 c p d ; p=0.006$ at $18 c p d)$ between normal and diabetic groups).

\section{SCANNING LASER OPHTHALMOSCOPY}

To assess retinal arteriovenous passage time nine diabetic subjects and 11 control subjects underwent scanning laser video fluorescein angiography during normal conditions and then during isocapnic hyperoxia created by breathing $100 \%$ oxygen. The two conditions were randomised and the subjects and technical staff masked to this order. Carbon dioxide was added during hyperoxic exposure to render control and high oxygen conditions isocapnic. ${ }^{11}$ Subjects breathed from a mouthpiece connected to a low resistance, two way breathing valve. ${ }^{11}$ On the inspired side, $100 \%$ oxygen was added to a mixing chamber, to which small amounts of carbon dioxide were added for isocapnia. ${ }^{9}$ This protocol raises end tidal partial pressure of oxygen $\left(\mathrm{Po}_{2}\right)$ to 600-700 $\mathrm{mm} \mathrm{Hg}$ while leaving end tidal partial pressure of carbon dioxide $\left(\mathrm{PCO}_{2}\right)$ constant at $35-40 \mathrm{~mm} \mathrm{Hg} .{ }^{11}$ Both of the end tidal gases were monitored by rapid response specific gas analysers placed in the initial portion of the expired air stream. ${ }^{11}$ Hyperoxia (or normoxia) was maintained for 15 minutes

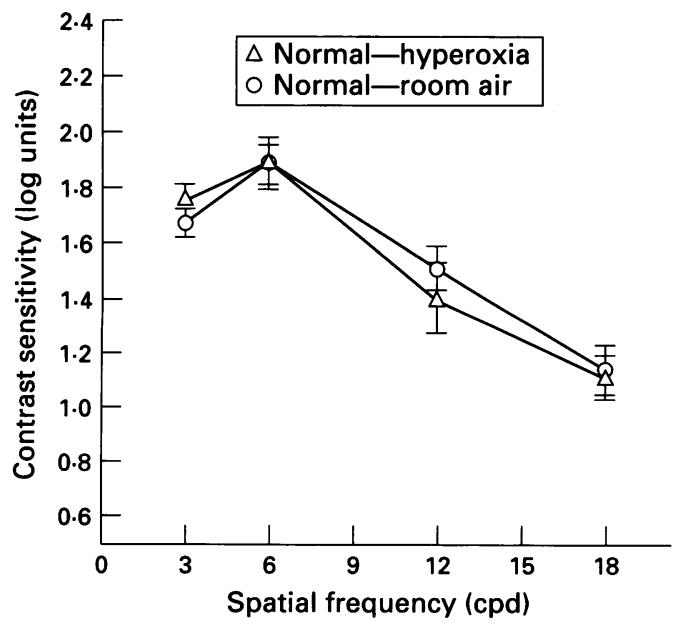

Figure 2 Contrast sensitivity at four spatial frequencies in normals breathing room air or $100 \%$ oxygen. Isocapnic hyperoxia failed to alter contrast sensitivity at any frequency. before fluorescein angiography and contrast sensitivity measurements were carried out. Arteriovenous passage time was measured by computer from digitised angiograms by calculating the time between the dye appearance (defined as attainment of $10 \%$ maximum dye intensity) on the temporal superior artery and the corresponding temporal vein. ${ }^{12}$

\section{CONTRAST SENSITIVITY}

Contrast sensitivity was assessed in all 20 subjects during normal conditions and during isocapnic hyperoxia created by breathing $100 \%$ oxygen. The two conditions were randomised and the subjects were masked to their order.

Contrast sensitivity was assessed with the CSV-1000 (VectorVision; Dayton, OH, USA) contrast testing instrument. This instrument has recently been demonstrated to provide the necessary test/retest stability for assessing disease related changes in contrast sensitivity. ${ }^{13}$ The instrument provides a retroilluminated translucent chart at a standardised light level of $85 \mathrm{~cd} / \mathrm{m}^{2}$. Four spatial frequencies (3, 6,12 , and $18 \mathrm{cpd}$ ) are tested using an orientation free two-alternative quasi-forced choice procedure. Testing time required is approximately 1 minute per eye.

\section{STATISTICAL ANALYSIS}

Unpaired $t$ tests were used to compare baseline or hyperoxic measurements in normal versus diabetic people. Paired $t$ tests were used to assess changes in each group as induced by hyperoxia; Bonferroni's correction was applied when multiple $t$ tests were performed using a single data set. ${ }^{14} \mathrm{~A} p$ value of $<0.05$ was regarded as statistically significant; all $t$ tests were two tailed.

\section{Results}

The insulin dependent diabetic patients and the normal controls were similar in mean age (23 (SD 8) years for patients; 26 (7) years for controls; $\mathrm{p}=\mathrm{NS}$ ), and in sex distribution (seven male, five female controls; six male, six female patients). Two hours before the study, blood glucose in the diabetic patients $(n=9)$ averaged $202(34) \mathrm{mg} / \mathrm{dl}$.

\section{ROOM AIR}

While breathing room air, diabetics displayed reduced contrast sensitivity at 12 and $18 \mathrm{cpd}$ compared with controls (Fig $1 ; p=0.003$ at 12 cpd, $\mathrm{p}=0.006$ at $18 \mathrm{cpd})$. Diabetic patients also demonstrated slowed arteriovenous passage time through the retina while breathing room air (Fig 6; $p=0.0001$ ).

\section{HYPEROXIA}

During the imposition of isocapnic hyperoxia, normal subjects showed no alterations in contrast sensitivity (Fig 2) while arteriovenous passage time was substantially slowed in these subjects (Fig 6; $\mathrm{p}=0 \cdot 0001$ ). In contrast, 
Figure 3 Contrast sensitivity at four spatial frequencies in diabetic patients with minimal retinopathy breathing room air or $100 \%$ oxygen. Isocapnic hyperoxia improved contrast sensitivity at 12 cycles per degree (asterisk indicates $p=0.042$ ).

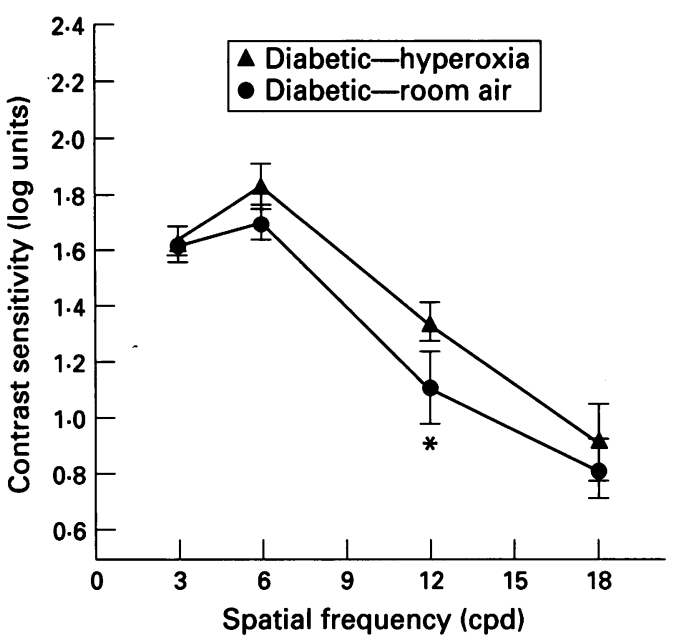

hyperoxia significantly improved contrast sensitivity in diabetic patients at $12 \mathrm{cpd}$ (Fig 3; $p=0.042$ ), without substantially altering arteriovenous passage time (Fig 6). Further, the improvement in contrast sensitivity at $12 \mathrm{cpd}$ in diabetic patients during hyperoxia eliminated the differences in contrast sensitivity between the two groups; no significant differences in contrast sensitivity were found between normals and diabetic patients in hyperoxia (Fig 4). Similarly, hyperoxia eliminated the differences between normals and diabetic patients in arteriovenous passage time seen during room air breathing (Fig 6).

The improvement in contrast sensitivity at $12 \mathrm{cpd}$ with hyperoxia in diabetic patients was proportional to the initial decrement in contrast sensitivity (Fig 5; $r=+0.84 ; \mathrm{p}=0.0008$ ). Impairment was determined relative to the mean sensitivity at $12 \mathrm{cpd}$ as measured in the present control group.

\section{Discussion}

In this study we confirmed that insulin dependent diabetic patients with minimal retinopathy exhibit both visual (reduced contrast sensitivity) and retinovascular (slowed arteriovenous dye passage) abnormalities. When hyperoxia

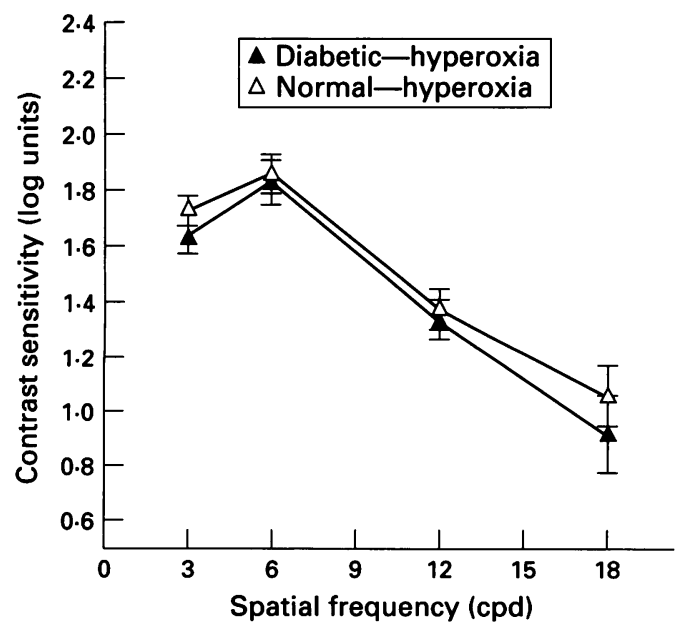

Figure 4 Contrast sensitivity at four spatial frequencies in normal people and diabetic patients with minimal retinopathy during isocapnic hyperoxia in both groups. No statistically significant differences in contrast sensitivity existed between the groups during hyperoxia.

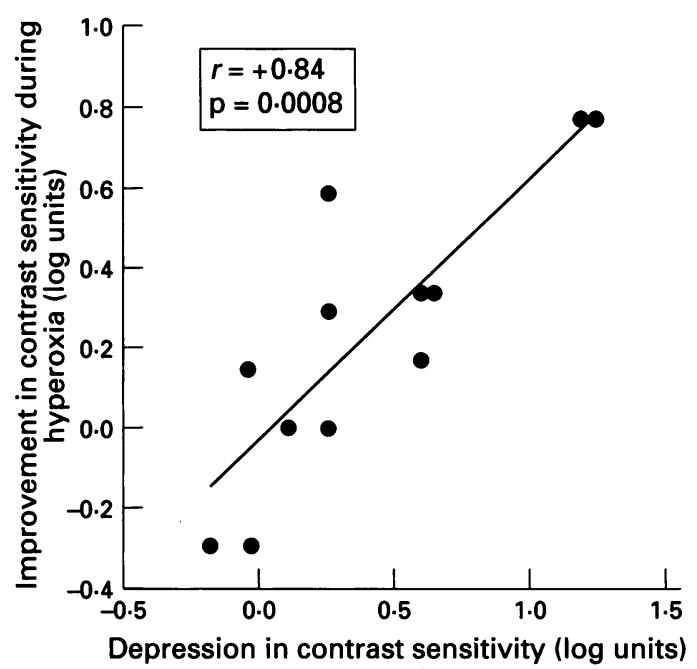

Figure 5 Significant positive correlation in diabetic patients of contrast sensitivity improvement during hyperoxia ( $y$ axis) with initial contrast sensitivity depression (x axis). All sensitivities were measured at 12 cycles per degree. Initial sensitivity depression was measured relative to the normal mean $(n=12 ; r=+0.84 ; p=0.0008)$.

was imposed upon these patients, contrast sensitivity significantly improved at $12 \mathrm{cpd}$ to levels indistinguishable from normal, even as arteriovenous passage time was unchanged. This result in patients contrasts with that found in healthy people, in whom hyperoxia did not alter contrast sensitivity even as it substantially slowed arteriovenous passage time.

The retinal vascular and visual defects caused by diabetes have been hypothesised to result from either localised tissue hypoxia ${ }^{89}$ or from hyperglycaemic pseudohypoxia. ${ }^{7}$ According to the latter hypothesis, cytosolic $\mathrm{NADH} / \mathrm{NAD}^{+}$ratios, elevated in true hypoxia, are also elevated when hyperglycaemia results in excess reduction of glucose to sorbitol (via aldose reductase) and the subsequent excessive oxidation of sorbitol to fructose. ${ }^{7}$ The resulting redox imbalance mimics that caused directly by low $\mathrm{Po}_{2}{ }^{7}$ If either theory is correct, then delivering more oxygen to a hyperglycaemic tissue should correct either low tissue $\mathrm{Po}_{2}$ or the redox imbalance and improve tissue function. ${ }^{7}$

We found both that hyperoxia improved mean contrast sensitivity in a group of diabetic patients, and that the improvement in sensitivity was proportional to the initial impairment. This result suggests that, at least in diabetic patients with minimal retinopathy, some aspects of diminished visual function remain acutely reversible. That these changes were seen at $12 \mathrm{cpd}$, and not so clearly, at $18 \mathrm{cpd}$, probably results from the smaller variance of repeated measures obtained at $12 \mathrm{cpd} .{ }^{13}$ The result also suggests that some factor linked to retinal tissue hypoxia (or pseudohypoxia) causes the contrast sensitivity decline seen early in the course of insulin dependent diabetes. Earlier studies using the pattern electroretinogram hypothesised that diabetes mellitus may affect the larger retinal ganglion cells most severely, ${ }^{15}$ although more generalised effects at every retinal neurosensory cell could not be ruled out. ${ }^{15}$ The mechanism linking diabetes to retinal neurosensory cell dysfunction is 


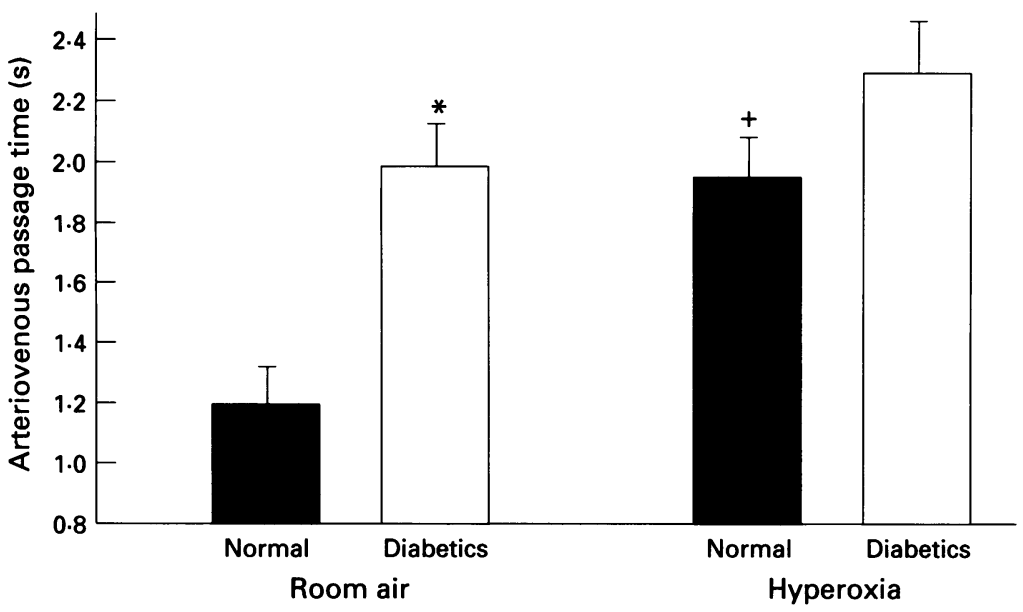

Figure 6 Retinal arteriovenous passage time in normal people and diabetic patients with minimal retinopathy under room air and hyperoxic conditions. While breathing room air, diabetics displayed slower arteriovenous passage of fluorescein dye (asterisk indicates $p=0.0001$ versus normals). Imposition of isocapnic hyperoxia failed to substantially change passage time in diabetics, while it did significantly slow transit in normals relative to the room air condition (plus indicates $p=0.0001$ versus room air for normals).

unknown, but could include the following sequence: (1) hyperglycaemia increases $\mathrm{NADH} / \mathrm{NAD}^{+}$ratios via a series of oxidation/ reduction reactions on glucose; (2) the consequent overactivation of aldose reductase reduces $(\mathrm{Na}, \mathrm{K})$ ATPase and $(\mathrm{Ca}, \mathrm{Mg})$ ATPase activities; and (3) reduced ATPase activities cause defects in neural conduction or sensitivity. ${ }^{715}$ This line of speculation is strengthened by the finding that these ATPase activities are reduced in retinal endothelial cells in experimental diabetes, ${ }^{16}$ and that aldose reductase inhibition in diabetic tissues restores ATPase activities to normal. ${ }^{17}$ Unfortunately, treatment of diabetic humans with aldose reductase inhibitors has thus far been somewhat disappointing. ${ }^{18}$ Certainly any comprehensive theory of the biochemical mechanism linking diabetes to defective visual function must also potentially include many other factors, such as changes in lipid metabolism, superoxide anion products, and the role of nitric oxide. ${ }^{719} 20$ Along this same line, it will be important to determine how fluctuations in blood glucose (in this study only measured 2 hours before testing) affect contrast sensitivity and blood flow.

Biochemical changes consequent to diabetes also result in vascular dysfunction. ${ }^{21}$ Retinal tissue oedema, capillary leakage, vascular sclerosis, and frank under or overperfusion are all well known consequences of chronic insulin dependent diabetes. ${ }^{1222} 23$ It remains debatable, however, to what extent vascular changes occur early in disease, before the emergence of frank retinopathy. For example, some authors find unchanged retinal blood flow in diabetics with only mild retinopathy ${ }^{24}$; others find early evidence of hyperaemia ${ }^{22}$ or of altered capillary perfusion. ${ }^{25}$ Our finding of slowed arteriovenous passage time in diabetic patients with minimal retinopathy, but with visual function impairment, confirms the presence of simultaneous vascular and visual dysfunction. Whether such vascular changes are essential for depression of contrast sensitivity remains unknown. It is also important to note that arteriovenous passage time is only partly dependent on bulk retinal blood flow: many factors, both blood flow and non-blood flow dependent, affect its value. ${ }^{721}$ Consequently, the slower arteriovenous passage time exhibited by diabetic patients could be created entirely by variables independent of flow, such as tissue blood volume or vascular permeability. ${ }^{7} 2123$

When healthy tissue is perfused with blood with increased oxygen content, blood flow decreases to allow total oxygen delivery to remain constant. ${ }^{11}$ This response, seen in the healthy retina, ${ }^{11}$ probably slowed arteriovenous dye passage in hyperoxia in this study in healthy individuals. ${ }^{11}$ These hyperoxia induced changes in retinal haemodynamics in healthy eyes left contrast sensitivity unchanged. On the other hand, earlier work suggests that diabetes blunts or abolishes hyperoxia induced vasoconstriction in affected tissues: retinal blood flow diminishes less than normal, or not at all, in diabetic patients breathing $100 \%$ oxygen. ${ }^{8}$ Our finding that there was no change in retinal arteriovenous passage time in diabetic patients given $100 \%$ oxygen to breathe may indicate a blunted vasoconstrictor or autoregulatory capacity in that tissue. However, as before, the indirect nature of the measurement makes it impossible to determine whether blood flow, or tissue oedema or permeability factors, are altered by hyperoxia in diabetes. What our data do indicate is that the haemodynamic response to an autoregulatory challenge is abnormal in diabetic patients with minimal retinopathy, providing further evidence that vascular abnormalities are present early in disease.

In conclusion, we have demonstrated reversibility (via $100 \%$ oxygen breathing) of the contrast sensitivity defects seen early in the course of diabetic retinopathy, and the association of these defects with retinal haemodynamic abnormalities. That an increase in arterial oxygen content can restore visual function in these people testifies both to the potential reversibility of early visual deficits in diabetes, and to the role of hypoxia (or hyperglycaemic pseudohypoxia) in their initiation.

Supported by NIH grant EY 10180-01 and an unrestricted grant from Research to Prevent Blindness, Inc.

$\mathrm{AH}$ is a recipient of the William and Mary Greve Award from Research to Prevent Blindness.

1 Saraux H. Contrast sensitivity and diabetes. Bull Soc Ophtalmol Fr 1990; 90: 465-8.

2 DiLeo MA, Caputo S, Palsini B, Porciatti V, Minnella A, Graco AV, et al. Nonselective loss of contrast sensitivity in visual system testing in early type I diabetes. Diabetes Care 1992; 15: 620-5.

3 Carr $\mathrm{R}$. Hue discrimination and $\mathrm{S}$ cone pathway sensitivity in early diabetic neuropathy. Invest Ophthalmol Vis $S c i$ 1990; 31: 1008-115.

4 Boschi D, Frosini MC, Mencucei R, Sodi A. The influence of early diabetes on the pattern electroretinogram. Doc Ophthalmol 1989; 71: 369-73.

5 Ghafour IM, Foulds WS, Allan D, McClure E. Contrast sensitivity in diabetic subjects with and without retinosensitivity in diabetic subjects with and
pathy. Br $\mathcal{F}$ Ophthalmol 1981; 66: 492-5.

6 Hyvärinen L, Laurinen P, Rovamo J. Contrast sensitivity in evaluation of visual impairment due to diabetes. Acta Ophthalmol 1983; 61: 94-101.

7 Williamson JR, Chang K, Frangos M, Hasan KS, Ido V, Kawamura $\mathrm{T}$, et al. Hyperglycemic pseudohypoxia and diabetic complications. Diabetes 1993; 42: 801-13.

8 Grunwald JE, Riva CE, Brucker AJ, Sinclair, SH, Petrig BL. Altered retinal vascular response to $100 \%$ oxygen breathing in diabetes mellitus. Ophthalmology 1984; 91: $1447-52$. 
9 Fallon TJ, Maxwell DL, Kohner EM Autoregulation of retinal blood flow in diabetic retinopathy measured by the blue light entoptic technique. Ophthalmology 1987; 94: 1410-5.

10 Diabetic Retinopathy Study Report 7. A modification of the Airlie House classification of diabetic retinopathy. Invest Ophthalmol Vis Sci 1981; 21: 210-26.

11 Harris A, Arend O, Kopecky K, Caldemeyer K, Wolf S, Sponsel $\mathrm{W}$, et al. Physiological perturbation of ocular and cerebral blood flow as measured by scanning laser ophthalmoscopy and color Doppler imaging. Surv ophthalmoscopy and color

12 Wolf S, Arend O, Toonen H, Bertram B, Reim M. Measurement of retinal micro- and macrocirculation in patients with diabetes mellitus. Clin Vis Sci 1991; 7: 461-9.

13 Pomerance G, Evans D. Test/retest reliability of the CSV1000 contrast test and its relationship to glaucoma therapy. Invest Ophthalmol Vis Sci 1994; 35: 3357-61.

14 Snedecor GW, Cochran WG. Statistical methods. Ames, Iowa: Iowa State University Press, 1980: 116

15 DiLeo MAS, Falsini B, Caputo S, Ghirlanda G, Porciatti V, Greco AV. Spatial frequency-selective losses with pattern electroretinogram type I (insulin-dependent) diabetic patients without retinopathy. Diabetologia 1990; 33: 726

.16 Lee T-S, MacGregor LC, Fluharty SJ, King GL Differential regulation of protein kinase $C$ and $(\mathrm{Na}, \mathrm{K})$ adenosine triphosphatase activities by elevated glucose levels in retinal capillary endothelial cells. f Clin Invest 1989; 83: 90 .

17 Sutera SP, Chang K, Marvel J, Williamson JR. Concurren increases in regional hematocrit and blood flow in diabetic rats: prevention by sorbinil. Am f Physiol 1992; 263: rats: prevent
H945-50.
18 Van Gerven JM, Tjon-A-Tsien AM. The efficacy of aldose reductase inhibitors in the management of diabetic complications. Comparison with intensive insulin treatment and pancreatic transplantation. Drugs Aging 1995; 6: 9-28.

19 Kern TS, Kowlurn RA, Engerman RL. Abnormalities of retinal metabolism in diabetes or galactosemia: ATPases retinal metabolism in diabetes or galactosemia: ATPases and gluta

20 Greene DA, Lattimer SA. Action of sorbinil in diabetic peripheral nerve: relationships of polyol (sorbitol) pathway inhibition to a myo-inositol mediated defect in sodium-potassium ATPase activity. Diabetes 1984; 33: 712-7.

21 Feke GT, Buzney SM, Ogasawara H, Fujio N, Goger DG, Spack NP, et al. Retinal circulatory abnormalities in type I diabetes. Invest Ophthalmol Vis Sci 1994; 35: 2968-75.

22 Grunwald JE, Riva CE, Sinclair SH, Brucker AJ, Petrig BL. Laser Doppler velocimetry study of retinal circulation in diabetes mellitus. Arch Ophthalmol 1986; 104: 991-6.

23 Grunwald JE, Riva CE, Baine J, Brucker AJ. Total retinal volumetric flow rate in diabetic patients with poor glycemic control. Invest Ophthalmol Vis Sci 1992; 33: 356-60.

24 Patel V, Rassam S, Newsom R, Wiek J, Kohner, E. Retinal blood flow in diabetic retinopathy. $B M F$ 1991; 305: 678-83.

25 Sinclair, SH. Macular retinal capillary hemodynamics in diabetic patients. Ophthalmology 1991; 98: 1580-4. 